\title{
Fazeres dos enfermeiros em uma unidade de internação psiquiátrica de um hospital universitário
}

\author{
Actions of nurses in a psychiatric hospitalization unit at a university hospital
}

Acciones de los enfermeros en una unidad de internación psiquiátrica de un hospital universitario

\section{Maria de Lourdes Custódio Duarte', Agnes Olschowsky'}

' Universidade Federal do Rio Grande do Sul, Escola de Enfermagem. Porto Alegre-RS, Brasil.

Submissão: 20/10/2010 Revisão: 13/9/2011 Aprovação: 12/10/2011

\begin{abstract}
RESUMO
A assistência de enfermagem em saúde mental vem passando por um processo de transformação de paradigma, do modelo asilar para o psicossocial. Temos o objetivo de identificar os fazeres dos enfermeiros em uma unidade de internação psiquiátrica em um hospital universitário. Pesquisa exploratória, descritiva, com abordagem qualitativa. Na coleta de dados, foi utilizada a entrevista semiestruturada com os enfermeiros. Os resultados mostram a apreensão de novos conceitos que orientam a assistência em saúde mental, na qual acolhimento, cuidado integral e individual e consideração da subjetividade dos usuários, norteiam essa prática. Os fazeres dos enfermeiros estão permeados por aspectos que vão além do biológico, incluindo a família e a sociedade.
\end{abstract}

Descritores: Enfermagem; Enfermagem Psiquiátrica; Saúde mental; Hospitais universitários.

\begin{abstract}
Nursing care in mental health has been undergoing a process of paradigmatic change, from the asylum model to the pychosocial support. The aim of this study is to identify nurses' practices in a psychiatric ward in a university hospital. An exploratory and descriptive study of qualitative approach was conducted. Semi-structured interviews were used to collect data. The results showed the apprehension of new concepts to guide mental health care: embracement, comprehensive care and individual consideration of the users' subjectivity guided nurses' practices. These practices are permeated by issues that go beyond the biological aspects, and include family and society.
\end{abstract}

Key words: Nursing; Psychiatric Nursing; Mental health; University hospitals.

\section{RESUMEN}

Cuidados de enfermería en salud mental ha sido objeto de un proceso de paradigma del modelo de asilo para el apoyo psicosocial. Nuestro objetivo es identificar los hechos de las enfermeras en una unidad psiquiátrica en un hospital universitario. Pesquisa exploratoria, descriptiva y cualitativa. En la recopilación de datos, utilizamos entrevistas semi-estructuradas con el personal de enfermería. Los resultados muestran la aprehensión de nuevos conceptos que guían el cuidado de la salud mental, donde la acogida, la atención integral y la consideración individual de la subjetividad de los usuarios guían esta práctica. Las prácticas de las enfermeras están atravesadas por cuestiones que van más allá de lo biológico, incluyendo familia y la sociedad. Palabras clave: Enfermería; Enfermería Psiquiátrica; Salud mental; Hospitales universitarios. 


\section{INTRODUÇÃO}

A assistência de enfermagem em saúde mental vem passando por um processo de transformação, tendo como desafio implementar a mudança de paradigma do modelo asilar para o modelo psicossocial. Essa transformação teve início no Brasil na década de 70 , em meio a um movimento político chamado Reforma Psiquiátrica,

A Reforma Psiquiátrica direciona o olhar para o sujeito como um ser pleno de subjetividades e visa a implementação de serviços extra-hospitalares, tendo como ênfase a assistência do sujeito no território, objetivando a superação do manicômio enquanto espaço de segregação, tutela e de isolamento. A proposta desse movimento é de reduzir leitos psiquiátricos, inserir os pacientes crônicos institucionalizados em programas comunitários e desenvolver equipamentos de saúde que possam substituir a internação psiquiátrica tradicional ${ }^{(1)}$.

Os manicômios, que faziam parte do modelo pré-reforma psiquiátrica, passaram a ser substituídos por alternativas comunitárias da rede básica de saúde, como os Centros de Atenção Psicossociais (CAPS), unidades básicas de saúde, ambulatórios especializados, hospital-dia, serviços de urgência e emergência psiquiátricas, leito ou unidade em hospital geral e serviços residenciais terapêuticos ${ }^{(2)}$. Essas alternativas ao manicômio, preconizadas pelo Ministério da Saúde, visam à inclusão social e à desinstitucionalização do sujeito em sofrimento mental.

No contexto do manicômio, o fazer do enfermeiro restringia-se ao de assistente, observando, vigiando, repreendendo e registrando o comportamento dos pacientes ${ }^{(3)}$. Esse modo de fazer tem base na formação flexneriana, reducionista, sob a idéia de que é preciso isolar para conhecer, e conhecer para intervir $^{(4)}$. A forma de tratar, nesse modelo, direciona o olhar apenas para o sintoma e para o diagnóstico, em um processo fragmentário do sujeito, incapacitando o profissional de estabelecer uma relação de ajuda com o usuário do serviço de saúde, tornando-o um objeto a ser tratado.

Os recursos, utilizados pela enfermagem no modelo hospitalocêntrico, eram de fazer o doente sentir medo, além de usar métodos punitivos e técnicas disciplinares, em que tratamento e punição se confundiam ${ }^{(5)}$. O controle da liberdade de expressão, do direito de ir e vir, de escolher, de decidir e de participar no tratamento era tolhido pela Enfermagem e demais profissionais. O objetivo terapêutico desse modelo era a adaptação da pessoa com patologia psiquiátrica aos padrões morais aceitáveis pela sociedade.

No modelo psicossocial, é necessário que o enfermeiro evolua para um papel terapêutico e não opressor, desprendendo-se do modelo médico que visa apenas a doença e não o sujeito em seu contexto social, considerando, assim, a pessoa com transtorno psiquiátrico como um sujeito na sua totalida$\mathrm{de}^{(6)}$. Na atualidade, o profissional deve organizar seus fazeres fundamentado na integralidade da atenção em saúde, em uma ótica mais flexível e criativa, vislumbrando um cuidado complexo e singular.

Nessa perspectiva de intervenção ampliada, as ações dos enfermeiros devem constituir-se em fazeres que olhem a pessoa dentro de seu contexto de vida, ou seja, alguém com história, desejos, relações, modos de viver com família e viver na cidade, no qual o seu cuidado aconteça a partir de suas demandas e necessidades. Essa transformação na saúde mental pressupõe a transformação de paradigmas na atenção aos sujeitos em sofrimento psíquico, substituindo a palavra "tratar" que leva uma nomeação diagnóstica, por "cuidar", termo que inclui várias dimensões da complexidade do sujeito(4).

A prática atual da Enfermagem Psiquiátrica deve ser pautada na noção de cuidado, como uma ação complexa e integral, respeitando e acolhendo as necessidades de cada indivíduo. Assim, o cuidado pressupõe capacidade para a escuta e o diálogo, além de disponibilidade para perceber o outro, como um sujeito com potencialidades, resgatando-lhe a autonomia e estimulando-lhe a cidadania.

A noção de integralidade da atenção ocorre pelo reconhecimento de que cada pessoa é um todo indivisível e social, que as ações de promoção e recuperação da saúde não podem ser fragmentadas ${ }^{(7)}$. Essas ideias corroboram o modelo psicossocial, que salienta que as interações entre profissionais e usuários dos serviços de saúde devem ser pautadas na horizontalidade, colocando esses dois protagonistas no mesmo patamar de importância no processo de reabilitação.

No campo da saúde mental e da psiquiatria, o enfermeiro utiliza a formação do vínculo terapêutico como principal ferramenta de trabalho. Esse processo de ajuda ao sujeito em sofrimento psíquico implica o conhecimento teórico associado à capacidade de comunicação e de autoconhecimento do enfermeiro. Essa capacidade de ajuda exige alguns requisitos básicos, como capacidade para amar, empatia pelo outro, capacidade técnica, científica e de consciência crítica ${ }^{(8)}$.

Desse modo, entendemos que o relacionamento é um dos eixos das diretrizes atuais da assistência psiquiátrica, visando à promoção da saúde mental. Pensamos que os fazeres do enfermeiro devem ser embasados em acolhimento, interdisciplinaridade, responsabilidade, vínculo e na integralidade das ações em oposição à vigilância e à opressão propostas nos manicômios. A partir do modelo psicossocial, esse profissional deve assumir novos fazeres, no qual o enfermeiro deve visualizar que saúde e doença não são sinônimos de bem e mal, positivo ou negativo, mas que os fazeres devem se organizar a partir da responsabilização e das relações de cuidado centradas no acolhimento e produção de vínculos, questionando as ações intervencionistas, limitantes e estigmatizantes ${ }^{(8)}$. Para o enfermeiro, isso implica na busca e na análise contínua de seus fazeres, sendo necessário considerar a atenção em saúde mental na sua dimensão ampliada como produção de vida.

Nesse novo cenário de cuidado, a enfermagem deve considerar a subjetividade do indivíduo, valorizando-o como cidadão com direitos e deveres. O estímulo à cidadania faz-se no cotidiano dos serviços de saúde e no contexto da multidisciplinaridade e na intersetorialidade. Para lidar com problemas complexos, há que diversificar ofertas de maneira integrada e buscar articulação em outros setores. Consequentemente, os profissionais de saúde, dentre eles o enfermeiro, precisam articular-se nessa caminhada ${ }^{(7)}$. O que se quer são fazeres comprometidos, nos quais as práticas assistenciais orientam-se na 
busca da autonomia, reinserção e inclusão social, aumentando a contratualidade da pessoa com transtorno psiquiátrico.

Através da concepção ampliada de assistência, do modelo psicossocial, preconizada pela Reforma Psiquiátrica, interessa-nos analisar os fazeres dos enfermeiros psiquiátricos. Entende-se por fazeres as atividades, as ações e intervenções realizadas no seu cotidiano assistencial.

Assim, o presente estudo, baseado no Trabalho de Conclusão de Curso Saberes e Fazeres dos enfermeiros em uma unidade de internação psiquiátrica de um hospital universitário $^{(9)}$, objetiva contribuir para os conhecimentos e concepções dos fazeres dos enfermeiros, articulando teorias e práticas, propondo uma reflexão sobre a atuação deste profissional no contexto proposto pela Reforma Psiquiátrica.

\section{REFERENCIAL METODOLÓGICO}

Este estudo tem caráter exploratório descritivo, com abordagem qualitativa. A pesquisa qualitativa responde questões muito particulares. Ela trabalha com o universo de significados, motivos, aspirações, crenças, valores e atitudes, que corresponde a um espaço mais profundo das relações dos processos e dos fenômenos que não podem ser reduzidos à operacionalização de variáveis ${ }^{(10)}$.

A pesquisa foi realizada na Unidade de Internação Psiquiátrica de um hospital universitário. Participaram todos os oito enfermeiros da equipe de enfermagem dessa unidade, que concordaram em participar e assinar o termo de Consentimento Livre e Informado. O tipo da amostra foi intencional, por convite, ocorrendo nos meses de agosto a outubro de 2005, no próprio local de trabalho, por meio de entrevistas, as quais foram gravadas e posteriormente transcritas. A pesquisa teve como critério de exclusão os enfermeiros que estiveram em licença saúde ou em férias no período da coleta de dados. O instrumento utilizado na coleta de dados foi a entrevista semiestruturada consistindo de dois questionamentos: Como ocorre o seu cotidiano de trabalho? Quais são as atividades que você desempenha na Unidade de Internação Psiquiátrica?

A análise dos dados deu-se através das três etapas propostas por Minayo: ordenação dos dados, classificação dos dados, análise final ${ }^{(10)}$. A primeira etapa consistiu no mapeamento dos dados obtidos nas entrevistas, ou seja, transcrição, leitura e reeleitura do material, e organização dos relatos; já na classificação dos dados, foi realizada através de uma leitura exaustiva e repetida dos textos, estabelecendo interrogações com base na fundamentação teórica, buscando identificar o que aparece de relevante e as ideias centrais das falas dos entrevistados; por fim, pretendeu-se estabelecer articulações entre os dados e referenciais teóricos de pesquisa, respondendo ao objetivo proposto no estudo.

Foi utilizado o Termo de Consentimento Livre e Informado, em duas vias, de acordo com a Resolução 196/96 do ConseIho Nacional de Saúde, que aprova diretrizes e normas regulamentadoras de pesquisa envolvendo seres humanos ${ }^{(11)}$. Este estudo foi aprovado pelo Comitê de Ética em Pesquisa do referido hospital sob o protocolo 05-331.

\section{FAZERES DOS ENFERMEIROS}

A formação do enfermeiro para o cuidado, como prática profissional, tem início em 1860, na Inglaterra Vitoriana com Florence Nightingale, onde ocorreu a organização da equipe de enfermagem em Nurses e Lady-Nurses. Através dessa divisão social do trabalho, as atividades relacionadas ao cuidado eram fragmentadas, já que às ladies cabia o ensino e supervisão, e às nurses as tarefas manuais ${ }^{(12)}$. Na atualidade, o enfermeiro tem seus fazeres organizados em ações de cuidado direto ao paciente (atividades assistenciais) e cuidado indireto centrado nas atividades de gerência do cuidado (atividades administrativas).

Contrapondo-se à visão fragmentada do cuidado, na contemporaneidade, os fazeres devem ter o usuário no centro do cuidado, ou seja, devem ser usuário-centrados. Essa prática assistencial propõe um processo de trabalho multiprofissional, permeado por ações baseadas no acolhimento, vínculo, autonomização e resolução ${ }^{(13)}$. Desse modo, os fazeres do enfermeiro devem estar pautados pela integralidade da atenção e acolhimento, buscando um atendimento ampliado, proporcionando espaços de fala, escuta, escolhas em que a vida do sujeito deve direcionar o cuidado em saúde mental.

$\mathrm{O}$ acolhimento traz à tona a ideia de relação, de ouvir e dialogar que pressupõe uma atitude da equipe de comprometimento em receber, escutar e tratar de forma humanizada os usuários e suas necessidades, por meio de uma relação de mútuo interesse entre trabalhadores e usuários ${ }^{(14)}$. Por sua vez, a integralidade aponta para uma visão mais abrangente das necessidades dos sujeitos de que cuidamos. Essas idéias devem nortear os fazeres dos enfermeiros para que possam cuidar, buscando transformar os modos de viver e sentir dos indivíduos, em sofrimento psíquico, no seu cotidiano.

Os enfermeiros entrevistados trazem o conceito de integralidade da assistência e o relacionamento interpessoal como concepções norteadoras do seu fazer, propondo que, ao cuidar, cada pessoa é um todo indivisível, parte da sociedade e que as ações de saúde devem configurar-se em um sistema capaz de prestar assistência integral.

É o cuidar do todo, é ter a visão ampla. Não tem como cuidar de uma coisa só do paciente psiquiátrico, tu acabas te envolvendo e cuidando dele inteiro, do corpo, da cabeça, do sentimento, da família (E6).

Nos depoimentos, transparece uma visão ampliada do fazer, não cuidando apenas da parte que está doente, mas explorando, considerando a pessoa na sua subjetividade, na sua história e suas relações com a família e a rede de apoio. $\mathrm{O}$ atendimento integral vai além da formulação de um planejamento terapêutico, mas contempla a reorientação das relações entre o Estado e a Sociedade e o olhar para o sujeito-usuário dentro de uma lógica de atendimento que considere o cuidado nas mais diversas dimensões do ser humano ${ }^{(15)}$.

Nesse cenário, o enfermeiro deve considerar e entender os fatores sociais e familiares para traçar uma estratégia de intervenção, embora não seja uma tarefa fácil, tendo em vista 
o desenvolvimento de suas atividades que, por muitos anos, ocorreram apenas dentro da instituição psiquiátrica ${ }^{(16)}$. Nesse sentido, o enfermeiro deve organizar seus fazeres desinstitucionalizando suas ações para práticas nas quais as relações aparecem como eixos articuladores para atividades mais abrangentes de saúde, em uma construção política e social.

Outra característica do fazer, apontada pelos enfermeiros, refere-se à peculiaridade de que as ações de enfermagem devem ser individualizadas, buscando atender às necessidades terapêuticas de cada um, tendo como pressuposto que cada indivíduo é um ser único.

O plano terapêutico é tu poderes avaliar os sinais e sintomas que o paciente está apresentando e poderes traçar um cuidado para ele. Poder estimular atividades recreativas, poder dar dados de realidade. É traçar um cuidado para ele que seja individualizado, porque cada paciente vai ter um plano terapêutico diferente. (E3).

O plano de atendimento é uma forma de individualizar o cuidado, mas, para que isso aconteça, é preciso que o enfermeiro esteja atento às necessidades do sujeito em sofrimento psíquico. O plano terapêutico é uma ferramenta de trabalho que está em constante mudança juntamente com as necessidades do paciente, implicando em uma constante avaliação para direcionar os fazeres dos enfermeiros. Para tanto, deve-se ter claro que essa ação é contínua, requer compromisso diário para não se caracterizar como um processo normativo do fazer.

No modelo psicossocial, o enfermeiro deve entender e estimular a participação do sujeito na formulação do seu cuidado, tendo presente que o fazer com outro, em relação, implica em troca e negociação da equipe com o usuário frente aos diferentes interesses.

Nas falas dos enfermeiros, pode-se observar que o profissional enfermeiro mostra-se disponível para uma prática profissional interdisciplinar, estando aberto para discutir, debater, aprender com outras equipes, trocando informações e compartilhando pensamentos em prol do bem-estar do paciente.

Discutir em equipe o que é melhor para o paciente naquele momento, para aquela situação, discutindo sobre a melhor forma de cuidar. Dividir, quando tu não sabes algo, com o colega (E3).

Troca de ideias, de informações com as demais equipes de trabalho: equipe médica, de nutrição, serviço social, psicologia [...] (E5).

Nota-se que os entrevistados estão receptivos a trabalhar e a aprender com outros profissionais. Apontam que a interdisciplinaridade implica a troca, o compartilhamento, a integração das diferentes áreas exigindo do profissional interlocução e disponibilidade para repensar suas ações. A interdisciplinaridade é um desafio, pois reconhece a necessidade de diferentes olhares sobre um mesmo fazer que se caracterize como coletivo.

O fazer também está relacionado com a interdisciplinaridade, conceito surgido no século XX com o objetivo de superar o individualismo do conhecimento, propondo-se a transcender e a ampliar a visão de um mundo fragmentado ${ }^{(17)}$. $\mathrm{Na}$ interdisciplinaridade, o fazer não fica exclusivamente limitado ao campo biológico ou dentro da área de atuação de cada profissional. Desse modo, caracteriza-se pelo envolvimento com todos os profissionais, reconhecendo diferentes olhares, buscando ampliar as ações da equipe de enfermagem e de saúde.

A interdisciplinaridade propõe troca de idéias e pressupõe uma atitude diferente a ser assumida pelos profissionais, substituindo uma visão fragmentada do conhecimento para uma visão ampliada, transcendendo o saber unitário de um profissional específico ${ }^{(17)}$. Atualmente, é considerada como uma alternativa para se alcançar as inovações propostas pelo novo modo de atenção em saúde mental, o qual requer profissionais capazes de articular conhecimentos profissionais específicos com o de toda a rede de saberes envolvidos no sistema de saúde ${ }^{(18)}$.

Nesse sentido, o fazer integral, como ação dos enfermeiros, tem como desafio oferecer um cuidado desenvolvido em aliança, considerando as dimensões da vida e da pessoa, em que saúde é uma produção social, ou seja, é condicionada pelo ambiente, lazer, trabalho, renda, educação, acesso aos serviços de saúde e outros.

A complexidade do cuidado direto também transparece nos depoimentos, onde o seu fazer caracteriza-se como amplo, abrangente, implementando ações para atenção individual que considera o sujeito em sua potencialidade e fragilidade para com a sociedade, ou seja, trabalho, lazer, moradia são questões consideradas na promoção da saúde mental Assim, evidencia-se a preocupação dos entrevistados em ajudar seu cliente a realizar atividades simples do dia-a-dia, como alimentação, higiene e vestir-se, atividades da vida diária que o constituem como pessoa reconhecida na sociedade.

Auxiliar na alimentação, na vestimenta, organizar o material, o material de banho, de quarto, a cama, porque quando eles forem para casa eles terão que fazer isso. Então, a gente deve estimular que eles continuem fazer aqui dentro, ou se eles não estavam fazendo lá que voltem a fazer aqui dentro. [...] São atividades como, jogar sinuca, tomar chimarrão, montar quebra-cabeça, mexer no computador, fazer trabalhos manuais, fazer um roda de conversa, fazer as unhas. Isso tudo ajuda na reabilitação (E3).

Transparece a ideia do fazer, indo além do sintoma, na qual autonomia, cidadania e reabilitação são objetivos desse cuidado. A reabilitação é um processo que aumenta a capacidade do usuário de estabelecer trocas sociais, resgatando sua autonomia em casa e no seu contexto social. Esse conceito visa trabalhar com o sofrimento e com a fragilidade e não com a incapacidade, valorizando o vínculo, a escuta e o acolhimento.

O que se busca, para a autonomia do doente mental, é a dependência de um maior número de trocas $^{(19)}$. Os profissionais de saúde devem pautar seus cuidados em um conjunto de diferentes técnicas que possam trabalhar com as necessidades 
individuais e as oportunidades do contexto onde o sujeito em sofrimento mental se insere. Entende-se que a reabilitação acontece no cotidiano, desde hábitos para o cuidado pessoal até as questões de trabalho, buscando formas singulares de intervir, resgatando a cidadania do paciente psiquiátrico e não a simples restituição dos direitos formais, mas a construção dos direitos substanciais, como afetos, relações, habilitação, trabalho e recursos materiais ${ }^{(10)}$.

Outra característica apontada no fazer dos entrevistados é o processo de enfermagem, uma metodologia de trabalho que busca dinamizar o cuidado. Traz a importância do registro de suas intervenções e avaliações diárias, contribuindo com um cuidado terapêutico e orientando o trabalho em equipe. Os diferentes registros, como padrões de sono, autocuidado e hábitos pessoais, possibilitam uma assistência mais abrangente, abrindo caminho para ação conjunta que procura ser ampla.

Rever os nossos diagnósticos todos os dias para ver se os nossos cuidados se mantém ou se a gente tem que alterar. Todos os dias a gente vivencia ele de forma diferente. Então, a gente está constantemente avaliando isso (E3).

Os diagnósticos de enfermagem proporcionam à Enfermagem uma estrutura para a organização de sua ciência. É, no entanto, responsabilidade individual, de cada enfermeiro, a aplicação do diagnóstico de enfermagem com cautela e cuidado ${ }^{(20)}$. Os enfermeiros devem utilizar o processo de enfermagem no seu cotidiano de trabalho, pois o mesmo lhe facilita o planejamento e a execução de suas atividades assegurando a qualidade da assistência prestada.

Considera-se que o processo de enfermagem pode contribuir para a prática de enfermagem mais autônoma e com bases científicas, porém, como apontado anteriormente, o fazer não deve se orientar por uma postura técnica de atenção à doença. O processo de enfermagem deve organizar-se com foco na complexidade do fenômeno da loucura, tendo clareza da importância de que as ações devem estar relacionadas à clínica ampliada.

Nessas atividades, a equipe de enfermagem troca informações sobre as ações realizadas nas 24 horas de cuidado da área. Relatam verbalmente o que ocorreu, o que foi feito ou não, checam os cuidados prescritos. Ou seja, é um espaço/ momento em que o fazer pode ser avaliado, planejado, orientando as ações da equipe de enfermagem.

O meu cotidiano de trabalho começa com a passagem de plantão, vendo como foi o turno anterior, como estavam os pacientes. Após isso a gente vai nos quartos para ver como estão os pacientes e ver aqueles que estão mais difíceis, que precisam de uma maior atenção (E1).

A passagem de plantão serve para dar a continuidade no trabalho (E3).

Entre os fazeres, apontados pelos enfermeiros, a passagem de plantão aparece como a primeira atividade realizada no cotidiano de trabalho, dando continuidade ao processo de cuidar. É um momento de troca de informações, caracterizado como de extrema importância para ordenar os seus fazeres, organizando o cuidado direto e o cotidiano de trabalho.

Esse momento de troca de informações funciona como um plano diário de trabalho, priorizando ações que devem ser realizadas no decorrer do trabalho, proporcionando a identificação de problemas singulares à cada paciente, com o objetivo de planejar e executar medidas de enfermagem que possibilitem a eficácia do cuidado ao paciente ${ }^{(20)}$. A equipe de enfermagem deve estar atenta nesse momento para que o plano diário de trabalho seja planejado e executado objetivando a satisfação do usuário em relação às suas demandas de saúde.

$\mathrm{Na}$ passagem de plantão, acontece a transmissão de informações entre os profissionais que terminam e os que iniciam o período de trabalho. Abordam o estado dos pacientes, assistência prestada, intercorrências, pendências e situações referentes a fatos específicos da unidade de internação que merecem atenção $^{(20)}$. A passagem de plantão é um fazer que organiza, que dá continuidade ao trabalho, devendo considerar a subjetividade e a particularidade das ações para cada paciente.

Além do cuidado direto, os enfermeiros realizam atividades gerenciais, ou seja, de cuidado indireto. Nas falas dos entrevistados, percebe-se que a supervisão da equipe enfoca as atividades administrativas como escalas, folgas e férias, entre outras, associadas à observação, orientação e organização do trabalho em equipe de enfermagem (técnicos de enfermagem e enfermeiros).

Supervisiono os técnicos e fico muito presente no ambiente. Acho que é a melhor forma de trabalhar. Observo as horas extras, as escalas, vejo as folgas. Então, a gente tem que administrar isso também (E2).

Não tem como fugir da administração. A assistência também deve ser administrada. (E5)

A supervisão vem sendo caracterizada como uma função administrativa que envolve um processo dinâmico e democrático de integração e coordenação dos recursos humanos e materiais. Esse processo administrativo envolve planejamento, execução, avaliação, objetivando a melhora da prática assistencial, servindo como uma importante ferramenta para gerir e organizar o trabalho em saúde. Quando bem planejada e conduzida, possibilita intervenções que oferecem melhores respostas, do ponto de vista do atendimento de necessidades focais, repercutem benéfica e satisfatoriamente na organização como um todo ${ }^{(20)}$.

Dessa forma, os fazeres dos enfermeiros em uma unidade de internação psiquiátrica devem estar pautados nos pressupostos da Reforma Psiquiátrica e da Reabilitação Psicossocial, procurando articular o fazer com o saber, além de procipiciar um espaço para discussão/reflexão sobre o cuidado, no qual sentimentos, limites e dificuldades são considerados condicionantes das ações. Esse espaço deve ser aproveitado como possibilidade de troca e diálogo sobre dificuldades individuais e coletivas, na qual as vivências do fazer devem ser expressadas e refletidas, buscando assim, organizar, transformar e facilitar o trabalho da enfermagem. 


\section{CONSIDERAÇÕES FINAIS}

O modelo psicossocial de cuidado ao indivíduo em sofrimentos psíquico, alicerçado nos pressupostos da Reforma Psiquiátrica, deve orientar as práticas assistenciais dos profissionais de saúde, dentre eles, os enfermeiros. Nesse modelo de atenção, os fazeres tem a centralidade das necessidades das pessoas em sofrimento psíquico, em que há um direcionamento para cidadania e autonomia como pressupostos teóricos para o cuidado em saúde mental.

Os enfermeiros que participaram desse estudo têm seus fazeres pautados no atendimento integral, através de um trabalho de equipe, incentivando a participação dos sujeitos em sofrimento psíquico no seu tratamento, compartilhando experiências com as famílias e com a comunidade. Assim, observa-se a apreensão de novos conceitos que orientam a assistência em saúde mental, na qual acolhimento, cuidado integral e individual e consideração da subjetividade norteiam essa prática.

Visualizamos o enfermeiro, potencialmente, como um importante agente de mudanças; entretanto, essa potencialidade estará diretamente relacionada ao grau de consciência desses trabalhadores, ou seja, quanto mais consciente de seu papel de trabalhador, inserido num contexto social e de cidadão num sistema político, mais apto estará para eleger instrumentos de trabalho que visem o resgate dessa mesma condição de sujeito-cidadão às pessoas com transtornos mentais.

\section{REFERÊNCIAS}

1. Kantorski LP, Machado A, Oliveira CA. Centro de atenção psicossocial: reinventando o cuidado em saúde mental. Texto Contexto Enferm. 2000; 9(1): 233-43.

2. Brasil, Ministério da Saúde. Guia de saúde mental. Porto Alegre: Ministério da Saúde; 2001.

3. Oliveira AGB, Alessi NP. O Trabalho de enfermagem em saúde mental: contradições e potencialidades atuais. Rev. Latino-Am. Enfermagem. 2003;11(3):333-40.

4. Alves DS. Integralidade nas políticas de saúde mental. In: Pinheiro R, Mattos RA, organizadores. Os sentidos da integralidade na atenção e no cuidado à saúde. 6ed. Rio de Janeiro: IMS CEPESC ABRASCO; 2006.

5. Machado SC, Moreira MC, Stipp MAC, Simões LM, Oliveira RMP, Leite JL. Liderança em enfermagem psiquiátrica. Esc. Anna Nery. 2006;10(4):730-4.

6. Pinheiro R. As práticas do cotidiano na relação oferta e demanda dos serviços de saúde: um campo de estudo e construção da integralidade. In: Pinheiro, R, Mattos RA, organizadores. Os sentidos da integralidade na atenção e no cuidado à saúde. 6ed. Rio de Janeiro: IMS CEPESC ABRASCO; 2006.

7. Nasi C, Cardoso ASF, Schneider J, Olschowski A, Wetzel C. Conceito de integralidade na atenção em saúde mental no contexto da reforma psiquiátrica. Rev. Min. Enf. 2009; 13(1): 147-152.

8. Olschowsky A. O ensino de enfermagem psiquiátrica e saúde mental: análise da pós-graduação "Latu Sensu". [Tese]. Ribeirão Preto (SP): Escola de Enfermagem de Ribeirão Preto, Universidade de São Paulo; 2001. 233f. Doutorado em Enfermagem.

9. Duarte MLC. Saberes e fazeres dos enfermeiros de uma unidade de internação psiquiátrica de um hospital universitário. [Trabalho de Conclusão de Curso]. Porto Alegre (RS): Escola de Enfermagem, Universidade Federal do Rio Grande do Sul; 2006. 44f. Graduação em Enfermagem e Obstetrícia.

10. Minayo MCS. O desafio do conhecimento: pesquisa qualitativa em saúde. Rio de Janeiro: ABRASCO; 1994. 269 p.

11. Ministério da Saúde (BR), Conselho Nacional de Saúde, Comitê Nacional de Ética em Pesquisa em Seres Humanos.
Resolução n. 196, de 10 de outubro de 1996: dispõe sobre as diretrizes e normas regulamentadoras de pesquisa envolvendo seres humanos. Brasília (DF): MS; 1996. 24 p.

12. Souza ACC, Muniz Filha MJM, Silva LF, Monteiro ARM, Fialho AVM. Formação do enfermeiro para o cuidado: reflexões da prática profissional. Rev Bras Enferm. 2006; 59(6): 805-7.

13. Merhy EE. A perda da dimensão cuidadora na produção da saúde: uma discussão do modelo assistencial e da intervenção no seu modo de trabalhar a assistência. In: Campos CR, Malta DC, Reis AT, Santos AF, Merhy EE. Sistema Único de Saúde em Belo Horizonte: reescrevendo o público. Belo Horizonte: Xamã; 1998.

14. Takemoto MLS, Silva EM. Acolhimento e transformações no processo de trabalho de enfermagem em unidades básicas de saúde de Campinas. Cad. Saúde Pública. 2007; 23(2): 331-340.

15. Pinho LB, Kantorski LP, Saeki T, Duarte MLC, Souza J. A integralidade no cuidado em saúde: um resgate de parte da produção científica da área. Rev Eletrônica Enferm. 2007; 9(03): 835-846.

16. Duarte MLC, Souza J, Kantorski LP, Pinho LB. Diferentes abordagens à família em saúde mental presentes na produção científica da área. Rev. Min. Enf. 2007;11(1):66-72.

17. Vilela EM, Mendes IJM. Interdisciplinaridade e saúde: estudo bibliográfico. Rev. Latino-Am. Enfermagem. 2003;11(4):525-31.

18. Tavares CMM. A interdisciplinaridade como requisito para a formação da enfermeira psiquiátrica na perspectiva da atenção psicossocial. Texto contexto Enferm. 2005;14(3):403-410.

19. Cardoso TVM, Oliveira RMP, Loyola CMD. Um entendimento linear sobre a teoria de Peplau e os Princípios da Reforma Psiquiátrica Brasileira. Esc. Anna Nery. 2006;10(4):718-24.

20. Siqueira ILCP, Kurcgant P. Passagem de plantão: falando de paradigmas e estratégias. Acta paul. enferm. 2005; 18(4):446-51. 